\title{
Chapter 15 \\ Properties of Radioactive Cs-Bearing \\ Particles Released by the Fukushima \\ Daiichi Nuclear Power Plant Accident \\ and Trace Element Analysis
}

\author{
Kazuhiko Ninomiya
}

\begin{abstract}
A large amount of radionuclides were released into the environment by the Fukushima Daiichi Nuclear Power Plant (FNPP) accident. By this accident, radioactive cesium (Cs)-bearing particles were also released. Since these particles stably exist in the environment, the influence of long-term exposure to radiation is concerned if the particle is ingested in the human body. In this review, in order to evaluate the influence of radioactive Cs-bearing particles on the environment, studies on radioactive Cs-bearing particles are summarized. Radioactive Cs-bearing particles can be classified into several kinds depending on their elemental composition and radioactivity. Although the production and distribution of radioactive Cs-bearing particles have still been unclear, details of the situation inside the nuclear reactor at the accident can be investigated from the classification and investigation of their production.
\end{abstract}

Keywords Fukushima Daiichi Nuclear Power Plant · Radioactive Cs-bearing particles $\cdot$ Radioactivity analysis $\cdot$ Cs contamination inventory

\subsection{Introduction}

On March 11, 2011, a large magnitude 9.0 earthquake occurred in the Pacific Coast of the northeast region of Japan and an accident occurred at the Fukushima Daiichi Nuclear Power Plant (FNPP) due to the large tsunami generated by the earthquake. This accident was classified in the most serious category of nuclear accident by the International Atomic Energy Agency (level 7), and a large amount of radionuclides were released into the atmosphere and ocean [1]. In addition to measuring the air

\footnotetext{
K. Ninomiya $(\bowtie)$

Department of Chemistry, Graduate School of Science, Osaka University,

Toyonaka, Osaka, Japan

e-mail: ninokazu@chem.sci.osaka-u.ac.jp
} 
dose rate, the distribution of nuclides, such as iodine-131 $\left({ }^{131} \mathrm{I}\right)$, tellurium- $129 \mathrm{~m}$ $\left({ }^{129 m} \mathrm{Te}\right)$, silver- $110 \mathrm{~m}\left({ }^{110 \mathrm{~m}} \mathrm{Ag}\right)$ and radioactive cesium $\left({ }^{134} \mathrm{Cs}\right.$ and $\left.{ }^{137} \mathrm{Cs}\right)$ deposited in soil was measured, and the initial contamination status was determined [2].

In a nuclear accident, volatilized radionuclides leak from the damaged fuel body and are released into the environment and captured by aerosols in the atmosphere. The aerosol behavior changes according to wind direction, speed and other factors at the time of the release event from the nuclear reactor, greatly affecting the transportation process [3]. Sulfuric acid is the most abundant aerosol in the atmosphere resulting in the radionuclide being transported as a sulfuric acid aerosol form. For the FNPP accident, by analyzing the contents of ionic compounds and radioactivities in the atmospheric aerosol for various particle sizes, it was determined that the amount of radioactive Cs strongly correlates with the amount of sulfuric acid aerosol [4].

On the other hand, heterogeneous spot-like contamination was reported via autoradiography analysis using imaging plates of environmental samples such as plant, soil and atmospheric filters just after the accident [5, 6]. Heterogeneous spot-like contamination was never washed out by water and was not the result of soluble sulfuric acid aerosols but arose from particles with concentrated radioactivity. Radioactive Cs is strongly adsorbed by minerals such as mica and some heterogeneous contamination was identified as soil particles [7-9]. However, Adachi et al. [10] reported in 2013 the detection of insoluble radioactive particles with spherical shapes of several micrometers in diameter from air samples collected in 2011. SEM image of the insoluble particle and its elemental composition is shown in Fig. 15.1. The particles are called variously such as radioactive Cs concentrated particles, Cs particles, insoluble particles, radiocesium-bearing microparticles (CsPs) and radioactive Cs-rich microparticles (CsMPs). The author calls the particles "radioactive Cs-bearing particles" hereafter in this article. Radioactive Cs-bearing particles are quite different from the "hot particles" found in the Chernobyl NPP accident [11] and various radioactive Cs-bearing particles have been discovered in Fukushima Prefecture and neighboring areas to date.

In this review, the author summarizes the recently reported papers on radioactive Cs-bearing particles that have been commonly detected after the FNPP accident. In addition to the properties of radioactive Cs-bearing particles, we examine their trace element analysis, distribution in the environment, formation processes and prospects for future research.
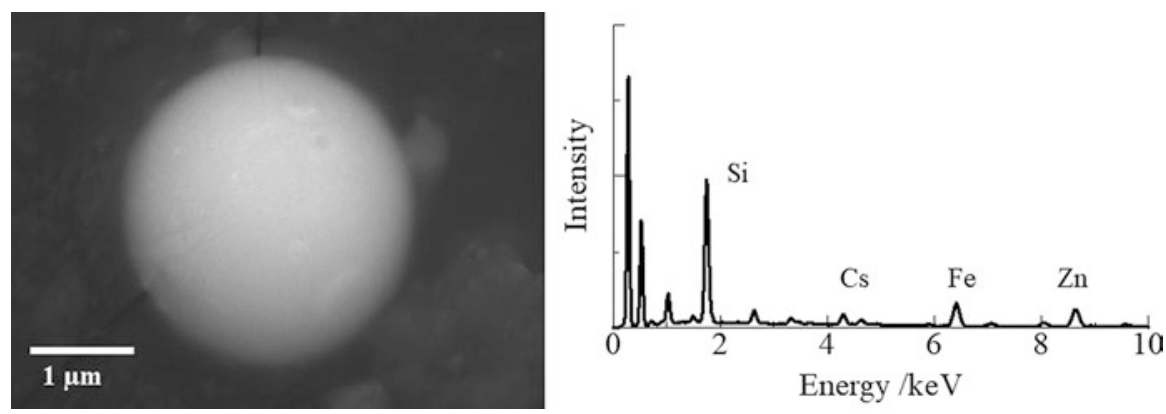

Fig. 15.1 SEM image of a radioactive Cs-bearing particle (left) and its EDS spectrum (right) 


\subsection{Properties of Radioactive Cs-Bearing Particles}

Radioactive Cs-bearing particles were isolated and analyzed for the first time from atmospheric samples (filters) collected in Tsukuba, $170 \mathrm{~km}$ southwest from FNPP [10]. From the scanning electron microscope (SEM) analysis, it is confirmed that radioactive Cs-bearing particles are spherical with a diameter of $2 \mu \mathrm{m}$ and contain $\mathrm{Cs}$, iron $(\mathrm{Fe})$, zinc $(\mathrm{Zn})$, manganese $(\mathrm{Mn})$ and chlorine $(\mathrm{Cl})$. Those radioactive Cs-bearing particles also contain several $\mathrm{Bq}$ of ${ }^{134} \mathrm{Cs}$ and ${ }^{137} \mathrm{Cs}$ with the particle radioactivity and size, indicating that a particle contains approximately $5 \mathrm{wt} \%$ of radioactive Cs. The particle is primarily composed of $\mathrm{SiO}_{2}$ though it is not clear because there are silicon ( $\mathrm{Si}$ ) background signals in the SEM analysis. They also reported radioactive $\mathrm{Cs-bearing}$ particles retain their shape even after washing with water. Abe et al. [12] conducted a more detailed analysis of radioactive Cs-bearing particles by synchrotron radiation X-ray (SR- $\mu$-X-ray) analysis showing that radioactive Cs-bearing particles contain molybdenum (Mo), barium (Ba), tin ( $\mathrm{Sn}$ ) and a trace amount of uranium (U). Furthermore, the chemical state of the metals contained in radioactive Cs-bearing particles was determined by SR- $\mu$-XANES (synchrotron radiation $\mathrm{X}$-ray absorption near edge structure) analysis, and Fe, Zn, Sn and Mo are present in their oxidized states as $\mathrm{Fe}^{3+}, \mathrm{Zn}^{2+}, \mathrm{Sn}^{4+}$ and $\mathrm{Mo}^{6+}$, respectively. Radioactive Cs-bearing particles can also be isolated from leaves, soil and dust in the high dose area of Fukushima Prefecture [13-15]. Sato et al. [16] performed detailed analysis of ${ }^{134} \mathrm{Cs}$ and ${ }^{137} \mathrm{Cs}$ radioactivity for radioactive Cs-bearing particles found in soil collected $20 \mathrm{~km}$ northwest of FNPP and reported the ${ }^{134} \mathrm{Cs} /{ }^{137} \mathrm{Cs}$ activity ratio just after the accident to be $1.03 \pm 0.01$. The authors also reported the clear linear relationship between particle size and radioactivity, that is, the specific activity is the same, indicating that radioactive Cs-bearing particles originate from the same source (Fig. 15.2).

Fig. 15.2 Relation between the size of a radioactive $\mathrm{Cs}$-bearing particle and its activity obtained by Sato et al. [16]

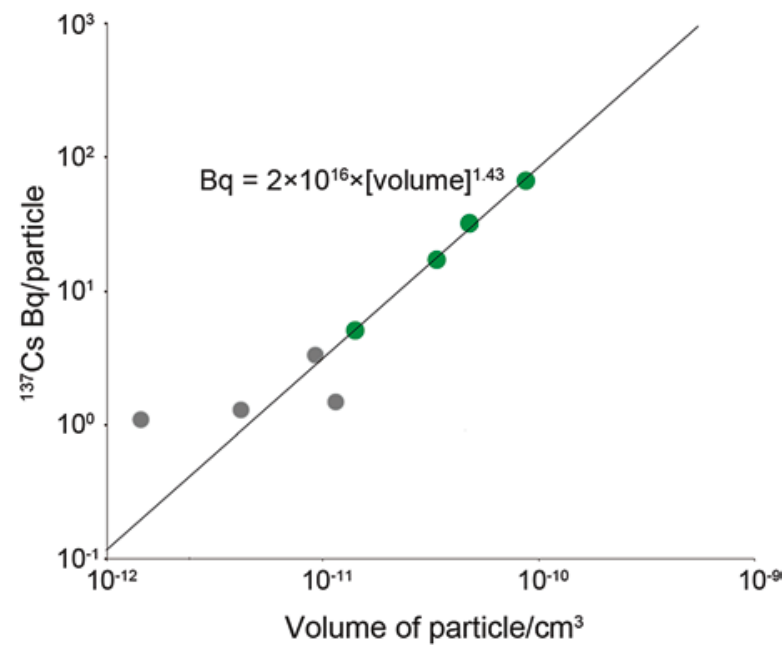


Table 15.1 Characteristics of radioactive Cs-bearing particles [15]

\begin{tabular}{l|l|l}
\hline \multirow{2}{*}{ Characteristic } & Particle type & B \\
\cline { 2 - 3 } Size distribution & A & $40-400 \mu \mathrm{m}$ \\
\hline${ }^{134} \mathrm{Cs} /{ }^{137} \mathrm{Cs}$ & $1-10 \mu \mathrm{m}$ & 0.93 \\
\hline Other radionuclides & 1.04 & Antimony-125 $(25 \mathrm{Sb})$ \\
\hline Distribution & N/A & Limited (North) \\
\hline Suspected emission date & Wide & March 12,2011 \\
\hline Source of reactor(s) & March 15,2011 & Unit 1 \\
\hline Specific radioactivity & Unit 2 or 3 & Low \\
\hline Elements in common & High & \multicolumn{2}{|l}{} \\
\hline Elements contained & $\mathrm{Si}, \mathrm{O}, \mathrm{Zn}$ & $\mathrm{Na}, \mathrm{Mg}, \mathrm{Ca}, \mathrm{Ba}$
\end{tabular}

Sato et al. [15] classified 19 radioactive Cs-bearing particles collected from soil and dust samples in Fukushima Prefecture and found two types of radioactive Cs-bearing particles depending on the discovery location (Table 15.1). Both radioactive Cs-bearing particles are primarily composed of $\mathrm{SiO}_{2}$ as a base material [17], but their detailed properties are different. The "type A" particles are similar to the particles found in Tsukuba [10,12]. These particles are characterized by several Bq of radioactivity per particle, but the specific activity is very large due to the small particle size, and typical radioactive Cs content is approximately several wt $\%$. The ${ }^{134} \mathrm{Cs} /{ }^{137} \mathrm{Cs}$ ratio was approximately 1 , so the source of the particle is likely Unit 2 or Unit 3, as estimated by Nishihara et al. [18]. The particles classified as "type B" have large activities, some exceeding $20 \mathrm{kBq}$ per particle. On the other hand, the specific activity is much smaller than that of type A particle because the size of the type $\mathrm{B}$ particle is typically several tens of $\mu \mathrm{m}$ or more. The ${ }^{134} \mathrm{Cs} /{ }^{137} \mathrm{Cs}$ ratio is approximately 0.9 , and the source of the type B particles is likely Unit 1 [18]. In addition, some type B particles exhibit Antimony-125 $\left({ }^{125} \mathrm{Sb}\right)$ activity in addition to radioactive Cs.

\subsection{Detailed Structure of Radioactive Cs-Bearing Particles by Destructive Analysis Method}

Yamaguchi et al. [13] and Kogure et al. [19] sliced radioactive Cs-bearing particles into a thin film using a focused ion beam (FIB) instrument and analyzed the film by transmission electron microscopy (TEM), X-ray microanalysis with scanning TEM (STEM) and energy-dispersive X-ray spectroscopy (EDS). These analyses clearly showed that radioactive Cs-bearing particles are primarily silicate glass composed of $\mathrm{Si}$ and $\mathrm{O}$. From the semiquantitative analysis by TEM of the entire particle, the content of $\mathrm{SiO}_{2}$ was estimated to be $70 \%$. It is also reported that the constituent elements are distributed almost uniformly in the radioactive Cs-bearing particle. However, the concentration of radioactive Cs outside the particles is approximately 
twice as high as the inside concentration, and potassium $(\mathrm{K})$ and rubidium $(\mathrm{Rb})$ are in the opposite distributions. The authors also reported that sulfide nanoparticles are implanted in the particles [13].

Furuki et al. [14] found that radioactive Cs-bearing particles contain fine pores $10-200 \mathrm{~nm}$ in size as determined by TEM analysis. In addition, they reported that crystalline nanostructures such as fine $\mathrm{CsOH}$ and $\mathrm{Fe}-\mathrm{Zn}$-oxide are formed inside the particles.

Imoto et al. [20] analyzed the isotope ratios of $\mathrm{U}, \mathrm{Cs}, \mathrm{Ba}, \mathrm{Rb}, \mathrm{K}$ and calcium (Ca) by secondary ion mass spectrometry (SIMS). They reported the ${ }^{235} \mathrm{U} /{ }^{238} \mathrm{U}$ isotope ratio to be 0.29 , which is significantly higher than the natural abundance of $U$ (0.00729). In SIMS analysis, it is difficult to distinguish between Cs and Ba isobars, but the isotopic ratio of $\mathrm{Ba}$ indicates that most $\mathrm{Ba}$ contained in radioactive $\mathrm{Cs}$-bearing particles originated from the decay of radioactive Cs. The ${ }^{87} \mathrm{Rb} /{ }^{85} \mathrm{Rb}$ isotope ratio also indicated that $\mathrm{Rb}$ derived from fission products. On the other hand, ratios of $\mathrm{K}$ and $\mathrm{Ca}$ were consistent with their respective natural isotopic ratios. The authors also attempted to measure the amount of ${ }^{90} \mathrm{Sr}$, but the concentration fell below the limit of detection. Ochiai et al. [21] reported that $U$ in radioactive Cs-bearing particles is present in iron oxide nanoparticles detected by high-angle annular darkfield scanning transmission electron microscopy (HAADF-STEM).

\subsection{Separating Radioactive Particles}

Radioactive Cs-bearing particles are very small and contain a $\mathrm{SiO}_{2}$ matrix. Many environmental particles have similar elemental compositions, so identification and extraction of radioactive Cs-bearing particles can be difficult. Although bulk extraction has not yet been developed, other separation methods have been developed to date, as discussed below.

Adachi et al. [10] succeeded in isolating radioactive Cs-bearing particles by fragmenting the sample. From autoradiography measurements using an imaging plate (IP), the position of radioactive Cs-bearing particles in the environmental sample was identified. They transfered the environmental sample including radioactive Cs-bearing particles to a carbon tape and separated it into small pieces. By repeating the visualization by IP and fragmentation of the carbon tape, the particle could be completely separated from other components in the environmental sample. Furuki et al. [14] developed a similar method for isolating the radioactive Cs-bearing particles from soil samples. The authors disperse the soil sample on grid paper and identify the position of radioactive Cs-bearing particles by autoradiography and then drop pure water on that position. The droplet is spreaded thinly and the position of the radioactive Cs-bearing particles is determined using IP again. By repeating this procedure, radioactive Cs-bearing particles are separated from the bulk soil sample.

Radiation measurement using a semiconductor or scintillation detector instead of autoradiography by IP is also used to separate radioactive Cs-bearing particles. 
Kurihara et al. [22] placed the environmental samples including radioactive Cs-bearing particles in a vial and divided each sample into two parts after mixing. After radiation measurement, water was added to the higher activity sample and subsequently divided into two samples. By repeating this procedure approximately 30 times, the radioactive Cs-bearing particles can be completely separated from the bulk soil.

\subsection{Environmental Fate of Radioactive Cs-Bearing Particles}

Ikehara et al. [23] conducted an autoradiography experiment with IP to determine the total amount of radioactive particles in soil samples. The soil was divided with a 114-mesh filter and the number of radioactive particles was determined by counting the spots of radioactivity on IP. The authors identified radioactive particles $(>0.06 \mathrm{~Bq})$ in surface soil collected from Okuma Town and Iitate Village and found 48-318 radioactive particles per gram of soil, and the ratio of total radioactivity of the particles to the entire soil sample was $8.53-31.8 \%$.

Igarashi et al. [24] reported the amount of radioactive particles in environmental samples by separating radioactive Cs-bearing particles from soil samples collected in Okuma Town, Fukushima Prefecture. Initially, the number of radioactive Cs-bearing particles (with radioactivity of $>1 \mathrm{~Bq}$ ) was identified by autoradiography and was separated using Kurihara's method [22]. The radioactivity ratio was approximately $0.2 \%$, differing from the results of Ikehara et al. [23]. This indicates that the proportion of radioactive Cs-bearing particles differs greatly depending on the sampling location.

Miura et al. [25] successfully isolated radioactive particles from suspended particles in rivers. From 2011 to 2016, suspended particles were collected by passing river water collected from the Kuchibuto River in Fukushima Prefecture through a filter. The authors isolated radioactive Cs-bearing particles found on the filter with a radioactivity more than $0.4 \mathrm{~Bq}$, and the activity ratio of radioactive Cs-bearing particles to the filter was estimated to be $0-46 \%$. The particles with weaker radioactivity $(0.1-0.4 \mathrm{~Bq})$ were identified via the IP analysis and total radioactivity on the filter was estimated to be $1.3-67 \%$.

Yamaguchi et al. [26] discussed the deterioration of radioactive Cs-bearing particles in the environment and determined the weathering of the particles and heterogeneity of the constituent $\mathrm{Cs}$ and $\mathrm{Rb}[13,19]$. The authors concluded that these phenomena are caused by several environmental factors including dissolution by cycling of wet and dry states as well as shape change caused by weathering kinetics of silicate glass. The lifetime of the particles is predicted to be shorter than several decades.

Okumura et al. [27] reported the stability of ${ }^{134} \mathrm{Cs}$ and ${ }^{137} \mathrm{Cs}$ by heating radioactive Cs-bearing particles. The authors conducted a heating experiment on six radioactive particles and showed that the radioactivity decreased at $600{ }^{\circ} \mathrm{C}$ and was completely lost when heated to $1,000{ }^{\circ} \mathrm{C}$. The size and characteristics of the heated 
particles were unchanged after heating. Upon heating, elements other than Cs, such as $\mathrm{Cl}$ and $\mathrm{K}$, were also lost as they were likely released from the particles in a similar manner. The authors also reported the heating experiment of radioactive Cs-bearing particles with soil samples and showed that the radioactivity in radioactive Cs-bearing particles moved to soil, causing significant dilution. They concluded that radioactivity in radioactive Cs-bearing particles can be decomposed by the treatment in incinerators with a burning temperature of approximately $900{ }^{\circ} \mathrm{C}$.

\subsection{Production of Radioactive Cs-Bearing Particles}

Radioactive Cs-bearing particles are not produced in the environment due to their high specific radioactivity and stability and are only produced in nuclear reactors and can be released by accidents. Determination of their production process is important to estimate the total amount of radioactivity released to the environment and to investigate the conditions in the nuclear reactor during the accident. Although the process of producing radioactive particles is unclear, several hypotheses have been proposed through detailed analysis of the produced radioactive particles.

The source of $\mathrm{Si}$ and $\mathrm{O}$, the main components of the particle, also remains unclear. Kogure et al. [19] showed that $\mathrm{SiO}_{2}$ was derived from concrete and fine particles of $\mathrm{SiO}_{2}$ which were formed by the molten core concrete interaction (MCCI) after the meltdown. Satou et al. [16] indicated that $\mathrm{SiO}_{2}$ is also contained in the heat insulating material with a lower melting point than that of concrete and is located near the nuclear reactor containment vessel. Kobata et al. [28] characterized the production of $\mathrm{CsFeSiO}_{4}$ under high temperature conditions by $\mathrm{CsOH}$ chemisorption to stainless steel containing $\mathrm{Si}$.

Kogure et al. [19] measured the diffusion coefficients of alkali metals and investigated that the diffusion coefficient of Cs is two orders of magnitude smaller than $\mathrm{K}$ or $\mathrm{Rb}$. The different distribution of alkali metals is derived from the various rates of diffusion of volatized alkali metals from the surface of the silicate particles. The authors also fabricated glass with the same composition in an attempt to reproduce the results of radioactive Cs-bearing particles. Imoto et al. [20] detected $\mathrm{Zn}-\mathrm{Fe}$ oxide nanoparticles in radioactive Cs-bearing particles, noting that iron nanoparticles are formed under high temperature conditions by MCCI and the evaporated $\mathrm{SiO}_{2}$ and $\mathrm{CsOH}$ can aggregate on nanoparticles. Ochiai et al. [21] pointed out that $\mathrm{U}$ is simultaneously taken into iron nanoparticles because $\mathrm{U}$ is found only in iron nanoparticles.

Fujita et al. [29] proposed a particle production process including the alkali melting reaction of $\mathrm{CsOH}$. It is well-known that radioactive $\mathrm{Cs}$ evaporated from the fuel body in the form of $\mathrm{CsOH}$, which is a strong base. The authors succeeded in producing particles by dissolving insulation materials or concrete mainly composed of $\mathrm{SiO}_{2}$ into droplets of $\mathrm{CsOH}$ melted at $300{ }^{\circ} \mathrm{C}$ as shown in Fig. 15.3. 

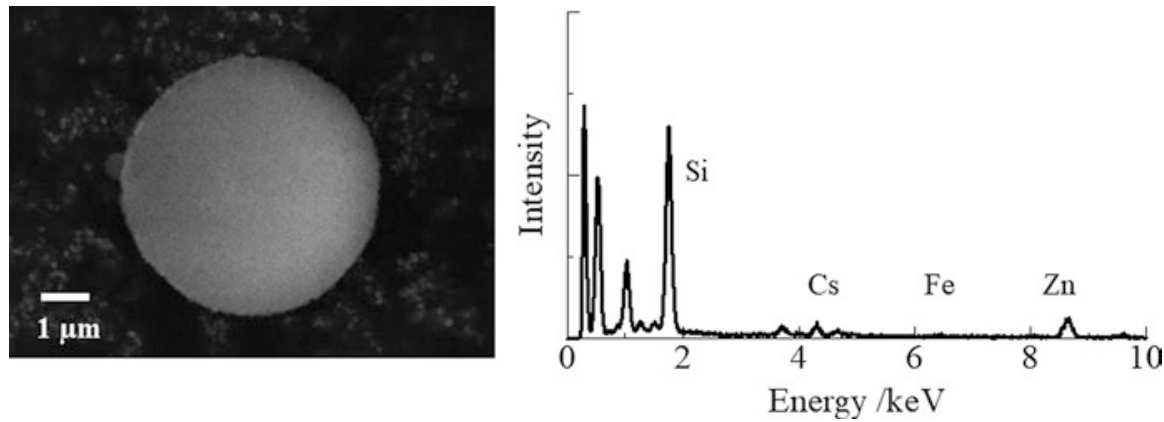

Fig. 15.3 SEM image of a reproducing radioactive Cs-bearing particle produced by the method of Fujita et al. [29] (left) and EDS spectrum of the reproducing particle (right)

\subsection{Future Study Prospects}

Herein, the author discussed the properties of concentrated radioactive particles found in the environment after the FNPP accident. These particles are insoluble in water and contain $\mathrm{SiO}_{2}$ as the main component. Although the production and distribution of radioactive Cs-bearing particles remain unclear, their detailed elemental composition is reported and classified into several distinct types of particles present in the environment. Studies related to particle classification and investigation of their production can clarify the release process of radionuclides at the time of the accident.

Acknowledgment The authors thank Dr. Satou (Japan Atomic Energy Agency) for providing data on the radioactive Cs-bearing particles. This work was partially supported by the grant the Nuclear Energy Science \& Technology and Human Resource Development Project (Through Concentrating Wisdom) \#281302 and the Grant-in-Aid for Scientific Research A \#16H01777 from the Ministry of Education, Culture, Sports, Science and Technology of Japan.

\section{References}

1. Chino M, Nakayama H, Nagai H et al (2011) Preliminary estimation of release amounts of ${ }^{131} \mathrm{I}$ and ${ }^{137} \mathrm{Cs}$ accidentally discharged from the Fukushima Daiichi Nuclear Power Plant into the atmosphere. J Nucl Sci Technol 48:1129-1134

2. Saito K, Tanihata I, Fujiwara M et al (2015) Detailed deposition density maps constructed by large-scale soil sampling for gamma-ray emitting radioactive nuclides from the Fukushima Daiichi Nuclear Power Plant accident. J Environ Radioact 139:308-319

3. Tsuruta H, Oura Y, Ebihara M et al (2014) First retrieval of hourly atmospheric radionuclides just after the Fukushima accident by analyzing filter-tapes of operational air pollution monitoring stations. Sci Rep 4:6717

4. Kaneyasu N, Ohashi H, Suzuki F et al (2012) Sulfate aerosol as a potential transport medium of radiocesium from the Fukushima nuclear accident. Environ Sci Technol 46:5720-5726 
5. Tanoi K, Hashimoto K, Sakurai K et al (2011) An imaging of radioactivity and determination of Cs-134 and Cs-137 in wheat tissue grown in Fukushima. Radioisotopes 60:317-322 (in Japanese)

6. Nakanishi TM, Kobayashi NI, Tanoi K (2013) Radioactive cesium deposition on rice, wheat, peach tree and soil after nuclear accident in Fukushima. J Radioanal Nucl Chem 296:985-989

7. Kogure T, Morimoto K, Tamura K et al (2012) XRD and HRTEM evidence for fixation of cesium ions in vermiculite clay. Chem Lett 41:380-382

8. Okumura T, Tamura K, Fujii E et al (2014) Direct observation of cesium at the interlayer region in phlogopite mica. Microscopy 63:65-72

9. Motai S, Mukai H, Watanuki T et al (2016) Mineralogical characterization of radioactive particles from Fukushima soil using $\mu-\mathrm{XRD}$ with synchrotron radiation. J Mineral Petrol Sci 111:305-312

10. Adachi K, Kajino M, Zaizen Y et al (2013) Emission of spherical cesium-bearing particles from an early stage of the Fukushima nuclear accident. Sci Rep 3:2554

11. Salbu B, Kerkling T, Oughton DH (1998) Characterization of radioactive particles in the environment. Analyst 123:843-850

12. Abe Y, Iizawa Y, Terada Y et al (2014) Detection of uranium and chemical state analysis of individual radioactive microparticles emitted from the Fukushima nuclear accident using multiple synchrotron radiation X-ray analyses. Anal Chem 86:8521-8525

13. Yamaguchi N, Mitome M, Akiyama-Hasegawa K et al (2016) Internal structure of cesiumbearing radioactive microparticles released from Fukushima nuclear power plant. Sci Rep 6:20548

14. Furuki G, Imoto J, Ochiai A et al (2017) Caesium-rich micro-particles: a window into the meltdown events at the Fukushima Daiichi Nuclear Power Plant. Sci Rep 7:42731

15. Satou Y, Sueki K, Sasa K et al (2018) Analysis of two forms of radioactive particles emitted during the early stages of the Fukushima - Nuclear Power Station accident. Geochem J 52:137-143

16. Satou Y, Sueki K, Sasa K et al (2016) First successful isolation of radioactive particles from soil near the Fukushima Daiichi Nuclear Power Plant. Anthropocene 14:71-76

17. Ono T, Iizawa $Y, A b e Y$ et al (2017) Investigation of the chemical characteristics of individual radioactive microparticles emitted from reactor 1 by the Fukushima Daiichi Nuclear Power Plant accident by using multiple synchrotron radiation X-ray analyses. Bunseki Kagaku 66:251-261 (in Japanese)

18. Nishihara K, Iwamoto H, Suyama K (2012) Estimation of fuel compositions in FukushimaDaiichi Nuclear Power Plant. JAEA-Data/Code 2012-018

19. Kogure T, Yamaguchi N, Segawa H et al (2016) Constituent elements and their distribution in the radioactive Cs-bearing silicate glass microparticles released from Fukushima nuclear plant. Microscopy 65:451-459

20. Imoto J, Ochiai A, Furuki G et al (2017) Isotopic signature and nano-texture of cesium-rich micro-particles: release of uranium and fission products from the Fukushima Daiichi Nuclear Power Plant. Sci Rep 7:540 9

21. Ochiai A, Imoto J, Suetake M et al (2018) Nuclear fuel fragments released to the environment with cesium-rich microparticles from the Fukushima Daiichi Nuclear Power Plant. Environ Sci Technol 52:2586-2594

22. Kurihara Y, Miura H, Higaki S et al (2017) Radioactive cesium-bearing particles in black substances, 2017 Annual Meeting of the Japan Society of Nuclear and Radiochemical Science

23. Ikehara R, Suetake M, Komiya T et al (2018) Novel method of quantifying radioactive Cesiumrich microparticles (CsMPs) in the environment from the Fukushima Daiichi Nuclear Power Plant. Environ Sci Technol 52:6390-6398

24. Igarashi J, Zhang Z, Ninomiya K et al (2018) Properties of the insoluble particles including radioactive Cs found in Okuma town, Fukushima prefecture. Proceedings of the 19th Workshop on Environmental Radioactivity. in press (in Japanese) 
25. Miura H, Kurihara Y, Sakaguchi A et al (2018) Discovery of radiocesium-bearing microparticles in river water and their influence on the solid-water distribution coefficient (Kd) of radiocesium in the Kuchibuto River in Fukushima. Geochem J 52:145-154

26. Yamaguchi N, Kogure T, Mukai H et al (2018) Structures of radioactive Cs-bearing microparticles in non-spherical forms collected in Fukushima. Geochem J 52:123-136

27. Okumura T, Yamaguchi N, Dohi T et al (2018) Loss of radioactivity in radiocesium-bearing microparticles emitted from Fukushima Daiichi nuclear power plant by heating. Sci Rep 8:9707

28. Kobata M, Okane T, Nakajima K et al (2018) Chemical form analysis of reaction products in Cs-adsorption on stainless steel by means of HAXPES and SEM/EDX. J Nucl Mater 498:387-394

29. Fujita N, Ninomiya K, Zhang Z et al (2016) Production simulation experiments of the insoluble Cs-concentrated particles released by the FDNPP accident and their imaging on a filter. Proceedings of the 17th Workshop on Environmental Radioactivity, KEK Proceedings 2016-8, $153-157$

Open Access This chapter is licensed under the terms of the Creative Commons Attribution 4.0 International License (http://creativecommons.org/licenses/by/4.0/), which permits use, sharing, adaptation, distribution and reproduction in any medium or format, as long as you give appropriate credit to the original author(s) and the source, provide a link to the Creative Commons license and indicate if changes were made.

The images or other third party material in this chapter are included in the chapter's Creative Commons license, unless indicated otherwise in a credit line to the material. If material is not included in the chapter's Creative Commons license and your intended use is not permitted by statutory regulation or exceeds the permitted use, you will need to obtain permission directly from the copyright holder.

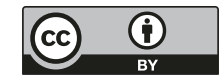

